\title{
Magnetic dynamics of weakly and strongly interacting hematite nanoparticles
}

\author{
Hansen, Mikkel Fougt; Bender Koch, Christian; Mørup, Steen
}

Published in:

Physical Review B Condensed Matter

Link to article, DOI:

10.1103/PhysRevB.62.1124

Publication date:

2000

Document Version

Publisher's PDF, also known as Version of record

Link back to DTU Orbit

Citation (APA):

Hansen, M. F., Bender Koch, C., \& Mørup, S. (2000). Magnetic dynamics of weakly and strongly interacting hematite nanoparticles. Physical Review B Condensed Matter, 62(2), 1124-1135.

https://doi.org/10.1103/PhysRevB.62.1124

\section{General rights}

Copyright and moral rights for the publications made accessible in the public portal are retained by the authors and/or other copyright owners and it is a condition of accessing publications that users recognise and abide by the legal requirements associated with these rights.

- Users may download and print one copy of any publication from the public portal for the purpose of private study or research.

- You may not further distribute the material or use it for any profit-making activity or commercial gain

- You may freely distribute the URL identifying the publication in the public portal

If you believe that this document breaches copyright please contact us providing details, and we will remove access to the work immediately and investigate your claim 


\title{
Magnetic dynamics of weakly and strongly interacting hematite nanoparticles
}

\author{
Mikkel F. Hansen \\ Department of Physics, Building 307, Technical University of Denmark, DK-2800 Lyngby, Denmark \\ Christian Bender Koch \\ Chemistry Department, The Royal Veterinary and Agricultural University, Thorvaldsensvej 40, DK-1871 Frederiksberg C, Denmark \\ Steen Mørup \\ Department of Physics, Building 307, Technical University of Denmark, DK-2800 Lyngby, Denmark
}

(Received 18 September 1998; revised manuscript received 7 December 1999)

\begin{abstract}
The magnetic dynamics of two differently treated samples of hematite nanoparticles from the same batch with a particle size of about $20 \mathrm{~nm}$ have been studied by Mössbauer spectroscopy. The dynamics of the first sample, in which the particles are coated and dispersed in water, is in accordance with the Néel expression for the superparamagnetic relaxation time of noninteracting particles. From a simultaneous analysis of a series of Mössbauer spectra, measured as a function of temperature, we obtain the median energy barrier $K_{\mathrm{Bu}} V_{\mathrm{m}} / k$ $=570 \pm 100 \mathrm{~K}$ and the preexponential factor $\tau_{0}=1.3_{-0.8}^{+1.9} \times 10^{-10} \mathrm{~s}$ for a rotation of the sublattice magnetization directions in the rhombohedral (111) plane. The corresponding median superparamagnetic blocking temperature is about $150 \mathrm{~K}$. The dynamics of the second, dry sample, in which the particles are uncoated and thus allowed to aggregate, is slowed down by interparticle interactions and a magnetically split spectrum is retained at room temperature. The temperature variation of the magnetic hyperfine field, corresponding to different quantiles in the hyperfine field distribution, can be consistently described by a mean field model for "superferromagnetism" in which the magnetic anisotropy is included. The coupling between the particles is due to exchange interactions and the interaction strength can be accounted for by just a few exchange bridges between surface atoms in neighboring crystallites.
\end{abstract}

\section{INTRODUCTION}

Hematite $\left(\alpha-\mathrm{Fe}_{2} \mathrm{O}_{3}\right)$, which is the most stable iron oxide, has the rhombohedral crystal structure isomorphous to that of corundum. ${ }^{1}$ In its bulk form it is antiferromagnetically ordered with the sublattice magnetization directions along the rhombohedral [111] axis below the Morin temperature $T_{\mathrm{M}} \approx 260 \mathrm{~K}$. Between $T_{\mathrm{M}}$ and the Néel temperature, $T_{\mathrm{N}}$ $\approx 956 \mathrm{~K}$, the spins lie in the (111) plane and are slightly canted away from antiferromagnetic orientation resulting in a weak ferromagnetic (WF) moment of about $0.4 \mathrm{~J} \mathrm{~T}^{-1} \mathrm{~kg}^{-1}$ in the (111) plane. Both the Morin transition temperature, the saturation magnetization and other magnetic properties depend on the particle size when the dimensions approach the nanometer range. Within the last few decades the magnetic properties of nanocrystalline hematite have therefore attracted considerable attention. ${ }^{2-12}$

Recently a sample of hematite nanoparticles with a size of about $16 \mathrm{~nm}$, prepared by heating of $\mathrm{Fe}\left(\mathrm{NO}_{3}\right)_{3} \cdot 9 \mathrm{H}_{2} \mathrm{O}$, was studied by magnetization measurements, $\mathrm{x}$-ray and neutron diffraction, and Mössbauer spectroscopy. ${ }^{12}$ The nanocrystalline hematite had a spontaneous magnetization of about $0.3-$ $0.4 \mathrm{~J} \mathrm{~T}^{-1} \mathrm{~kg}^{-1}$ which is only slightly enhanced compared to the value for polycrystalline bulk WF hematite $(0.3$ $\mathrm{J} \mathrm{T}^{-1} \mathrm{~kg}^{-1}$ ). It was found that the dominating contribution to the magnetization in the nanoparticles arises from the canting of the magnetic sublattices and that the magnetic energy of the nanocrystalline hematite sample in zero external field could be described by ${ }^{1,12}$ where $\vec{M}_{1}$ and $\vec{M}_{2}$ are the sublattice magnetizations, $\vec{D}$ is the Dzialoshinskii vector, and $\theta$ and $\phi$ are the angles between $\vec{M}_{1}-\vec{M}_{2}$ and the [111] direction and an easy direction in the basal (111) plane, respectively. The two first terms are the isotropic and anisotropic exchange interactions. The third and fourth terms are the anisotropy energies for rotation of $\vec{M}_{1}-\vec{M}_{2}$ out of the (111) plane and within the (111) plane, respectively. It was found that the sublattice magnetization directions were confined to the (111) plane $(\theta \approx \pi / 2)$ even in an applied magnetic field of $4 \mathrm{~T}$ and hence that $-K_{1}$ was much larger than all other anisotropy constants. The superparamagnetic relaxation sensed by Mössbauer spectroscopy was therefore of two-dimensional nature. From analyses of a temperature series of Mössbauer spectra using the Néel relaxation law for the superparamagnetic relaxation time

$$
\tau=\tau_{0} \exp \left(\frac{E_{b}}{k T}\right),
$$

it was found that the median energy barrier for a $180^{\circ}$ rotation of the sublattice magnetization vectors in the basal plane was $E_{\mathrm{bm}} / k=K_{\mathrm{Bu}} V_{\mathrm{m}} / k \approx 600 \mathrm{~K}$ and the preexponential factor was determined to $\tau_{0}=(6 \pm 4) \times 10^{-11} \mathrm{~s}$.

Studies of interparticle interactions between magnetically ordered nanoparticles have attracted both theoretical and experimental interest. ${ }^{6,7,13-30}$ This interest has so far mostly 
been focused on the properties of systems of ferro- and ferrimagnetic particles with pure dipole interactions, ${ }^{19-30}$ but it is interesting to compare the behavior of such systems with the behavior of systems of interacting antiferromagnetic or weakly ferromagnetic particles as the nature of the interaction may be different in these systems. For ferro- and ferrimagnetic particles both magnetic dipole-dipole interactions and exchange interactions may be important. For antiferromagnetic and weakly ferromagnetic particles, exchange coupling between the surface atoms of neighboring particles may be the most important interaction. ${ }^{14}$

In this paper, we compare two samples of weakly ferromagnetic hematite nanoparticles from the same batch. In one sample the particles were coated with surfactant molecules and suspended in water in order to minimize interparticle interactions. The other sample was prepared by washing and drying uncoated particles. Mössbauer spectra of the two samples are substantially different, but the studies suggest that the difference in strength of interparticle interactions can explain the observations. The magnetic dynamics of the coated particles is in accordance with the Néel expression for superparamagnetic relaxation whereas the behavior of the uncoated particles can be described in terms of the "superferromagnetism" model. ${ }^{13}$

\section{EXPERIMENTAL}

The sample was prepared by forced hydrolysis of Fe(III) ions at elevated temperature. First 0.02 mole of $\mathrm{Fe}\left(\mathrm{NO}_{3}\right)_{3}$ . $9 \mathrm{H}_{2} \mathrm{O}$ (Merck, p.a.) was added to $200 \mathrm{~cm}^{3}$ of distilled water at a temperature close to the boiling point. Next $1.3 \mathrm{~cm}^{3}$ of $25 \%$ ammonia solution was added under vigorous stirring and the solution was left in the reaction vessel at $100^{\circ} \mathrm{C}$ under reflux conditions for $2.5 \mathrm{~h}$. The resulting brownish red precipitate was separated from the solution by centrifugation and was washed twice in water and once in acetone. The as-prepared sample contained a disordered iron-containing phase in addition to hematite (see Sec. III A). In order to remove this phase, the sample was treated with oxalate. This treatment has proven effective to dissolve poorly crystalline iron oxides and hydroxides. ${ }^{31,32}$ The $\mathrm{pH}$ of a $0.2 \mathrm{M}$ solution of di-ammonia oxalate was adjusted to 3 by addition of $1 \mathrm{M}$ $\mathrm{HCl}$. About $250 \mathrm{~cm}^{3}$ of this solution per gram of the asprepared sample was added and the suspension was left in darkness for $24 \mathrm{~h}$ with stirring. The resulting red precipitate was then separated from the solution by centrifugation and washed twice in water.

One part of the oxalate-treated sample was washed in water and in acetone and was left for drying. This sample will be referred to as the dry sample. Another part of the oxalate treated sample was washed in water and was kept suspended in water. The $\mathrm{pH}$ of this suspension was increased to about 10 by addition of $25 \%$ ammonia solution and subsequently the surfactant (oleic acid) was added. The sample was then given an ultrasonic treatment resulting in a stable suspension of the particles. This sample will be referred to as the coated sample.

Transmission electron micrographs of both the coated and dry samples were obtained using a Philips EM 430 electron microscope operated at $300 \mathrm{kV}$. The samples were given an ultrasonic treatment in water and a droplet of each sample

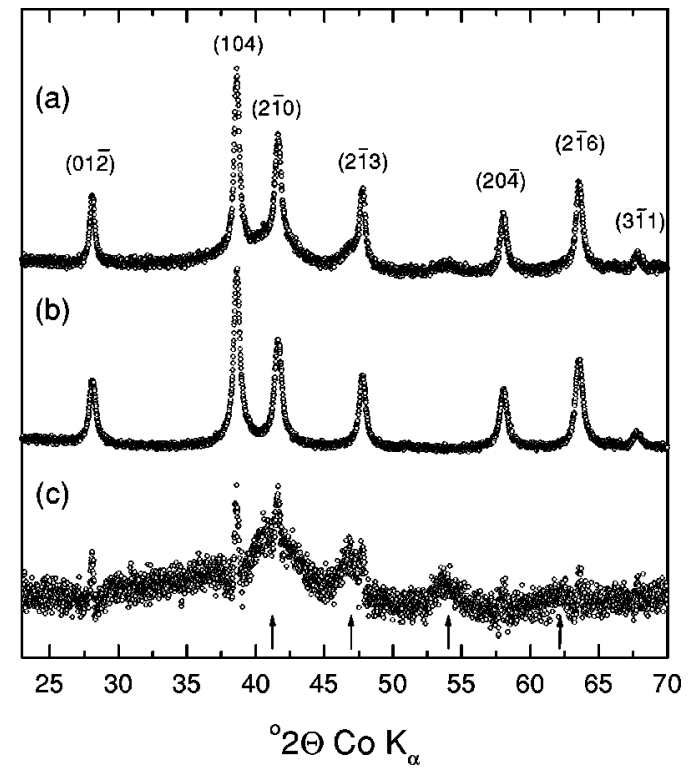

FIG. 1. X-ray diffraction spectra of (a) the as-prepared sample and (b) the dry sample. (c) Difference between the two spectra (scaled to same intensity of the sharp peaks).

was placed on a carbon coated copper grid and dried for the electron microscopy studies. X-ray diffraction (XRD) was performed on both the as-prepared sample and the dry sample in a Philips PW1050 diffractometer using the $K_{\alpha}$ radiation from a Co tube. The Mössbauer spectra were obtained with a conventional Mössbauer spectrometer in the constant acceleration mode using a $50 \mathrm{mCi}$ source of ${ }^{57} \mathrm{Co}$ in $\mathrm{Rh}$. The calibration was performed using a $12.5 \mu \mathrm{m}$ thick foil of $\alpha$-Fe at room temperature. All isomer shifts are given with respect to that of $\alpha$-Fe at room temperature. The Mössbauer spectra measured below $80 \mathrm{~K}$ were obtained using a closed cycle helium refrigerator from APD Cryogenics Inc. and the spectra obtained from $80 \mathrm{~K}$ to $295 \mathrm{~K}$ were obtained using a liquid nitrogen cryostat. Spectra measured above room temperature were obtained using a home-built furnace. A sample of commercial hematite powder (Merck, p.a.) was used as a reference for the variation of the saturation hyperfine field with temperature.

\section{RESULTS}

\section{A. Sample purity and morphology}

In Fig. 1 are shown XRD spectra of the as-prepared sample and the dry sample. The difference spectrum, obtained after normalization to eliminate the sharp diffraction lines, is shown in the lower part of the figure. The spectrum of the dry sample contains only reflections characteristic of hematite whereas the spectrum of the as-prepared sample has some additional features due to the phase removed by the oxalate treatment. These features are more clearly seen in the difference spectrum, which exhibits broad lines at ${ }^{\circ} 2 \Theta \approx 42$, 47,54 , and 64 . These four reflections are consistent with those reported in the same $2 \Theta$-interval for 6-line ferrihydrite. ${ }^{32}$

The broadening of the diffraction lines for both the asprepared sample and the dry sample was analyzed. No consistent effect of stress on the line broadening could be ob- 


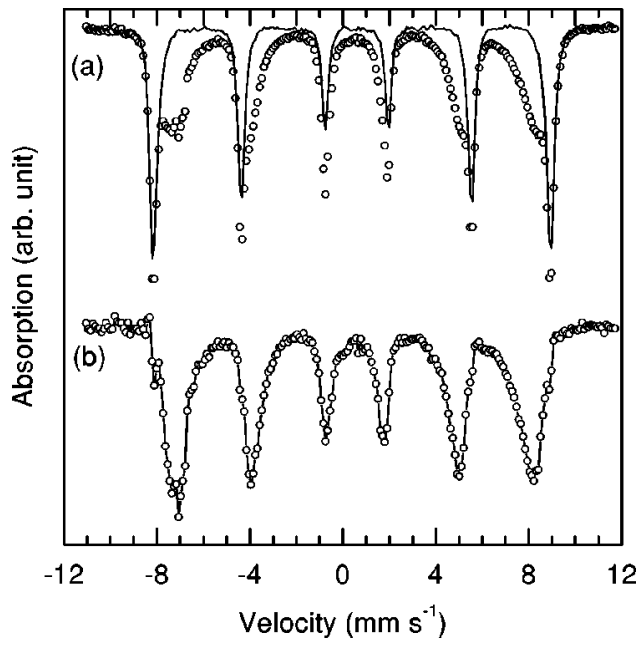

FIG. 2. (a) Mössbauer spectra of the as-prepared sample (open circles) and of the dry sample (data points connected by lines) obtained at $15 \mathrm{~K}$. (b) Difference between the two spectra obtained after scaling to eliminate the sextet due to hematite. The points are connected by lines for visual clarity.

served and it was therefore assumed that the broadening of the diffraction lines was solely due to the small crystallite size. In the difference spectrum in Fig. 1 some sharp spikes are seen at the positions of the diffraction peaks. This indicates that the hematite diffraction lines in the dry sample are slightly broader than those in the as-prepared sample. By use of the Scherrer formula, ${ }^{33}$ we estimated the crystallite dimensions $d=24 \pm 6 \mathrm{~nm}$ for the as-prepared sample and $d=20$ $\pm 4 \mathrm{~nm}$ for the dry sample. The larger uncertainty for the as-prepared sample is due to the presence of the lines from the ferrihydrite phase. This suggests that the particle dimensions have been slightly reduced by the oxalate treatment. The particle size estimated from the XRD spectrum of the dry sample is consistent with the observations made by electron microscopy. The electron micrographs of both the coated and dry samples showed roughly spherical particles. Detailed analyses of the size distributions were not possible due to agglomeration of the particles. No significant difference between the dry and coated samples could be observed.

The Mössbauer spectra of the as-prepared and dry samples obtained at $15 \mathrm{~K}$ are shown in Fig. 2. The difference between the two spectra, after normalization to eliminate the component with sharp lines, is also shown. The spectrum of the dry sample consists of a sextet with sharp Lorentzian shaped lines and a magnetic hyperfine field of $B_{\mathrm{hf}}=53.1$ $\pm 0.2 \mathrm{~T}$, an isomer shift of $\delta=0.49 \pm 0.01 \mathrm{~mm} \mathrm{~s}^{-1}$ and a quadrupole shift of $\epsilon=-0.10 \pm 0.01 \mathrm{~mm} \mathrm{~s}^{-1}$. These parameters are consistent with those expected for hematite in the absence of the Morin transition. ${ }^{1}$ The width of lines 1 and 6 was $0.39 \mathrm{~mm} \mathrm{~s}^{-1}$. Within the statistical uncertainty this spectrum was identical to the spectrum of the coated particles obtained at the same temperature. No traces of other components (such as goethite) could be found in these Mössbauer spectra. In the spectrum of the as-prepared sample there is an additional component which constitutes about $50 \%$ of the spectral area. This component is more clearly seen in the difference spectrum. Van der Kraan observed a similar component in Mössbauer spectra of hematite nanoparticles and attributed it to surface atoms. ${ }^{3}$ However, the fact that the component can be removed by the oxalate treatment shows that, at least in our samples, the component is not due to surface atoms of the hematite nanoparticles. The parameters corresponding to the peak positions of this component are $B_{\mathrm{hf}}=48.5 \pm 1.0 \mathrm{~T}, \delta=0.48 \pm 0.02 \mathrm{~mm} \mathrm{~s}^{-1}$, and $\epsilon=-0.05$ $\pm 0.02 \mathrm{~mm} \mathrm{~s}^{-1}$ and they correspond well to those reported for 6-line ferrihydrite. ${ }^{34,35}$

\section{B. Mössbauer spectra of the coated sample}

Mössbauer spectra of the coated sample were obtained at temperatures up to $240 \mathrm{~K}$. Higher temperatures lead to a significant reduction of the Mössbauer effect because the melting point of the ice matrix is approached. Representative spectra are shown in Fig. 3(a). At low temperatures, the spectra consist of a single sextet with sharp lines. As the temperature is increased, the lines become asymmetrically broadened towards the centroid of the spectrum, and a doublet appears. At intermediate temperatures, the sextet and doublet coexist, and at $240 \mathrm{~K}$ the spectrum is almost fully collapsed to a doublet. This behavior is typical for a sample containing noninteracting or weakly interacting superparamagnetic particles with a broad size distribution. The median blocking temperature, defined as the temperature at which $50 \%$ of the spectrum is magnetically split, is about $150 \mathrm{~K}$. A spectrum was also measured at $T=240 \mathrm{~K}$ in a magnetic field of $B=0.7 \mathrm{~T}$ applied perpendicular to the gamma-ray direction [Fig. 3(a), bottom]. It is seen that the applied field restores the magnetic splitting. This shows unambiguously that the collapse of the sextet is due to superparamagnetic relaxation of the particles and that the particles have a nonzero magnetic moment. A rough estimate of the magnetic moment, $\mu$, corresponding to the median particle size can be obtained from this spectrum using ${ }^{15,36}$

$$
\vec{B}_{\mathrm{obs}} \approx \vec{B}_{0}\left(1-\frac{k T}{\mu B}\right)+\vec{B},
$$

where $B_{\text {obs }}$ is the observed median hyperfine field and $B_{0}$ is the saturation hyperfine field. This equation assumes small anisotropy and a field-independent value of the magnetic moment. In the present case the magnetic moment depends on the external field due to the field dependence of the WF magnetic moment ${ }^{12}$ and the anisotropy may not be negligible. If both these effects are neglected, however, it leads to an overestimation of $\mu$. As the saturation hyperfine field at $240 \mathrm{~K}$ we use the hyperfine field from the measurement on the reference bulk hematite sample (after subtracting $0.8 \mathrm{~T}$ because $T<T_{\mathrm{M}}{ }^{1}$ ), $B_{0}=52.5 \mathrm{~T}$. From a hyperfine field distribution $\mathrm{fit}^{37}$ to the spectrum shown in the lower part of Fig. 3 (a), we obtain the median hyperfine field $B_{\mathrm{obs}} \approx 34 \mathrm{~T}$. Using $d=20 \mathrm{~nm}$ and Eq. (3), we obtain $\mu \approx 1500 \mu_{B}$ and hence that the value of the spontaneous magnetization, $\sigma_{s}=\mu / V \rho$, is smaller than $0.6 \mathrm{~J} \mathrm{~T}^{-1} \mathrm{~kg}^{-1}\left(\rho=5256 \mathrm{~kg} \mathrm{~m}^{-3}\right.$ is the density of bulk hematite). This implies that the magnetization of the hematite nanoparticles in the present study is similar to that of WF bulk hematite in agreement with the observations made by Bødker et al. ${ }^{12}$

\section{Mössbauer spectra of the dry sample}

Mössbauer spectra of the dry sample are shown in Fig. 3(b) for some of the measuring temperatures. The highest 

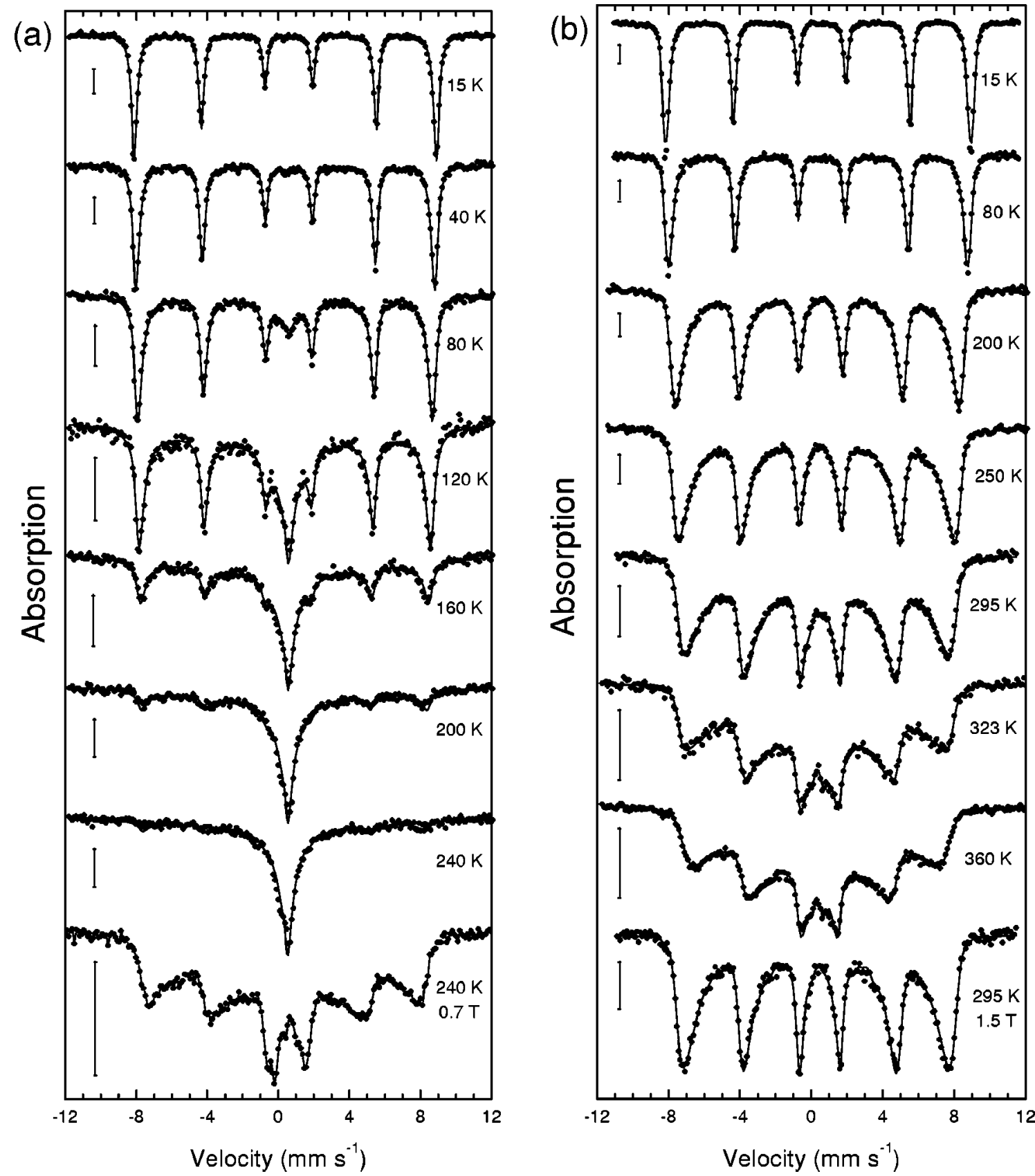

FIG. 3. (a) Mössbauer spectra of the coated sample obtained at the indicated temperatures. The lines in the zero-field spectra are the fits to the modified Blume-Tjon model described in the text. For visual clarity the points in the in-field spectrum are connected with lines. (b) Mössbauer spectra of the dry sample obtained at the indicated temperatures. The lines are fits to hyperfine field distributions. The vertical bars in the figure indicate $1 \%$ absorption in the spectra.

measuring temperature was $360 \mathrm{~K}$. A comparison of the spectra obtained at room temperature before and after the measurement at $360 \mathrm{~K}$ revealed that the magnetic hyperfine splitting was slightly better resolved after the heating. This is probably due to partial removal of adsorbed water from the particle surfaces. ${ }^{14}$ All measurements presented in the following were made after the $360 \mathrm{~K}$ measurement. The spectra of the dry sample are substantially different from those of the coated sample. At least up to $295 \mathrm{~K}$, the spectra consist only of magnetically split components, but especially for $T$ $\geqslant 200 \mathrm{~K}$, the lines are asymmetrically broadened towards the centroid of the spectrum, and even at room temperature there is no indication of a doublet in the spectrum. Application of a magnetic field of $1.5 \mathrm{~T}$ at room temperature results in a sharpening of the lines and an increased average hyperfine field [Fig. 3(b), bottom].

\section{DISCUSSION}

\section{A. Qualitative interpretation of the results}

Any model, which may be used to describe the behavior of the present samples must be able to explain two effects, namely the absence of fast superparamagnetic relaxation in the dry sample and the line broadening and the low values of the average hyperfine field in this sample above $200 \mathrm{~K}$. 
One might suggest that the apparent increase of the blocking temperature in the dry sample simply could be due to an increase in the superparamagnetic relaxation time which may occur because of, for example, an increase in the anisotropy energy constant. Such an effect could be related to the influence of adsorbed molecules on the surface anisotropy. Dormann et al. ${ }^{19,23,27}$ have suggested that interparticle interactions always result in an increase of the energy barrier in Eq. (2) and that $\tau_{0}$ also may be affected by interparticle interactions. The theoretical background for these suggestions is, however, questionable. ${ }^{28}$ In the spectra of the dry sample, obtained above $200 \mathrm{~K}$, lines 1 and 6 are broader than the inner lines, and even for a very substantial broadening of lines 1 and 6 there is no collapse of lines 3 and 4 as one would expect if the line broadening was due to conventional Néel relaxation, ${ }^{38}$ and the asymmetry is too large to be explained by collective magnetic excitations. ${ }^{36}$ Thus, the spectral shape is not in accordance with a model for superparamagnetic relaxation and another explanation is therefore needed. Since the particles in the two samples are identical, as they are taken from the same batch, it seems that the different behavior must be related to the difference in interparticle interactions.

The spectral shape of the dry sample at high temperatures $(T>200 \mathrm{~K})$ is very similar to that observed in samples of goethite $(\alpha-\mathrm{FeOOH})$ nanoparticles, and it is therefore relevant to consider the models that have been suggested to describe the properties of samples of nanocrystalline goethite. One possible explanation for the reduced hyperfine fields could be surface effects. ${ }^{39}$ Such effects can in fact be significant in Mössbauer spectra of very small particles, but for goethite and hematite particles with dimensions of the order of $20 \mathrm{~nm}$, only a small fraction of the atoms is expected to be influenced by surface effects, ${ }^{13}$ and it is therefore unlikely that surface effects can explain the present results.

It has been suggested that a mean field model for interacting particles can explain the results for goethite nanoparticles. ${ }^{13}$ The basic idea in this model is that interparticle interactions (exchange or dipole interactions) can lead to "super-ferromagnetic", ordering of the (sublattice)magnetization directions of the particles at temperatures at which the particles would exhibit fast superparamagnetic relaxation if they were noninteracting. The order parameter of the frozen "super-ferromagnetic" state varies with temperature in a way similar to that of, for example, ferromagnetic materials. This state, which should be understood in an abstract sense as the ordered state resulting from the interparticle interactions, does not necessarily imply a macroscopic ferromagnetic moment of the sample. In the model, it is assumed that the relaxation is fast, such that the line broadening due to relaxation is negligible, and the Mössbauer spectra should consist of sextets with a magnetic hyperfine splitting corresponding to the average hyperfine field. However, variations in strength of interparticle interactions and in magnetic anisotropy lead to different temperature dependencies of the magnetic hyperfine fields, and this is the reason for the line broadening. Studies of goethite particles with different interparticle interactions seem to support the "superferromagnetism" model. ${ }^{14,16,40}$ The model was, however, later criticized by Bocquet et al., ${ }^{41,42}$ who performed neutron diffraction, Mössbauer spectroscopy and magnetization measurements as a function of temperature on similar samples. Based on the disappearance of the magnetic peaks in neutron diffraction spectra, the collapse of the sextet in the Mössbauer spectra and the occurrence of a cusp of the zero field cooled magnetic susceptibility at approximately the same temperature, they concluded that the observed phenomena were due to a lower Néel temperature of the fine particles compared to bulk goethite rather than due to interactions between superparamagnetic particles.

Bocquet et ll $^{42}$ also estimated the magnetic anisotropy energy constant of goethite from the field dependence of Mössbauer spectra at low temperatures. Using the value estimated in this way they calculated a superparamagnetic blocking temperature, which was far above the temperatures at which the broadened spectra were measured, indicating that the assumptions behind the model for "superferromagnetism", were not fulfilled. However, the model used to calculate the field dependence of the Mössbauer spectra and the spin-flop field was based on the assumption of uniaxial anisotropy and a collinear antiferromagnetic structure, although the magnetic structure of goethite is much more complicated. ${ }^{43}$ Therefore the model seems to be too crude. Secondly, it has been shown that even a very small uncompensated magnetic moment in antiferromagnetic particles can result in a substantial increase of the spin-flop field, ${ }^{43,44}$ and small antiferromagnetic particles will inevitably have an uncompensated moment. Furthermore, the anisotropy was estimated at low temperatures, but at higher temperatures, at which the model is applied, the anisotropy may be smaller. An estimate of the anisotropy constant for ferritin, based on the field dependence of the Mössbauer spectra, yielded a blocking temperature of the order of 900 $\mathrm{K}^{45}$ Mössbauer spectroscopy measurements on the same sample, however, revealed that the blocking temperature was about $40 \mathrm{~K}$. Therefore, the estimates of the magnetic anisotropy of antiferromagnetic particles from the field dependence of Mössbauer spectra are at least in some cases inadequate.

Bocquet et al. ${ }^{42}$ proposed that the asymmetric broadening in the Mössbauer spectra can be explained by a model in which it is assumed that the particles consist of clusters created by a high concentration of vacancies. Furthermore, they assumed that the magnetization vectors in the clusters precess rapidly with a precession angle $\theta$. The magnetic hyperfine fields, observed in the Mössbauer spectra, were for $B$ $\leqslant B_{0}$ assumed to be given by $B=B_{0} \cos \theta$. The magnetic hyperfine field distribution was assumed to be proportional to the Boltzmann factor $p(B) \propto \exp \left(E_{c} \cos \theta / k T\right)$, where $E_{c}$ is the height of the potential barrier of the form $-E_{c} \cos \theta$ related to interactions between the clusters. Thus, it was assumed that the precession is fast compared to the time scale of Mössbauer spectroscopy, whereas the transition rate for transitions between the precession states, characterized by different $\theta$ values, is small. The latter assumption may not be correct for the following reason: If $\tau_{0}$ is considered as an attempt frequency for transitions across the energy barrier of a superparamagnetic cluster, one should expect that the relaxation between the precession states near an energy minimum will have a characteristic time scale of the same order as $\tau_{0}$, i.e., typically much smaller than the time scale of 


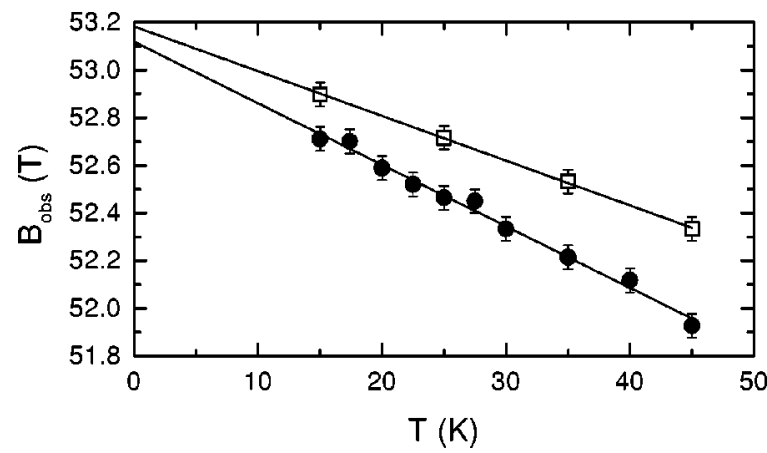

FIG. 4. Observed median magnetic hyperfine field for the coated (๑) and dry samples $(\square)$ as a function of temperature. The lines are fits in accordance with Eq. (4).

Mössbauer spectroscopy. Thus the "cluster model"' seems to be based on assumptions, which are not verified.

The interpretation of the results for goethite may still be an open question. In the present study we have directly measured the superparamagnetic blocking temperature of the non-interacting hematite particles, and we have found that essentially all particles have blocking temperatures below $250 \mathrm{~K}$. Thus, the assumption used in the "superferromagnetism" model is clearly fulfilled above this temperature, and there is no reason to introduce new parameters as in the model by Bocquet et al. ${ }^{42}$

The fact that the application of a magnetic field of $1.5 \mathrm{~T}$ at $295 \mathrm{~K}$ results in sharper lines in the Mössbauer spectra and an increase in the average hyperfine field shows that the line broadening is due to fluctuations of the sublattice magnetization vectors in the particles, which to some extent can be suppressed by the applied field. If the reduced values of the hyperfine field were due to a lowering of the Néel temperature, as it has been suggested for goethite, ${ }^{41}$ one would not expect such a dependence on the applied field. Therefore, the observed field dependence supports the validity of the "super-ferromagnetism" model for the dry hematite sample.

According to Bocquet's "cluster model," one should expect the same cluster dynamics in the coated and the dry samples at low temperatures. We have fitted the lowtemperature Mössbauer spectra of the two samples with distributions of magnetic hyperfine fields. ${ }^{37}$ In Fig. 4 we have shown the median hyperfine field, $B_{\text {obs }}(T)$, of the two samples for $T \leqslant 45 \mathrm{~K}$. For both samples $B_{\text {obs }}(T)$ has a linear temperature dependence, but the slope for the dry sample is significantly smaller than that for the coated sample. The reduction of the magnetic hyperfine field in nanoparticles compared to the bulk value is at low temperatures in general given by ${ }^{36}$

$$
B_{\text {obs }}(T) \approx B_{0}(T)\left[1-\frac{k T}{2 \kappa V}\right],
$$

where $B_{0}(T)$ is the temperature dependent hyperfine field in the absence of relaxation phenomena. [For hematite, the variation of $B_{0}(T)$ is small below $45 \mathrm{~K}$ compared to the observed temperature dependence of $B_{\text {obs }}$ ]. The value of $\kappa$ is related to the shape of the energy minimum and depends on both the anisotropy energy constants and interparticle interactions. $^{13,36}$ The different slopes observed for the two samples in Fig. 4 can be explained by the models for collec- tive magnetic excitations and "super-ferromagnetism.,"13,36 This will be discussed in more detail in the subsequent sections. The qualitative analysis of the data thus indicates that the most adequate model for description of the properties of the dry sample is the "super-ferromagnetism" model.

\section{B. Analysis of the spectra of the coated particles}

At low temperatures, the variation of the median magnetic hyperfine field of the coated particles is in accordance with Eq. (4). The solid line in Fig. 4 is a fit to Eq. (4) which yielded $\kappa V / k=1030 \pm 100 \mathrm{~K}$. The variation of $B_{0}(T)$ with temperature was assumed to follow that of bulk hematite after subtracting $0.8 \mathrm{~T}$ for $T<T_{\mathrm{M}} \cdot{ }^{1}$ For the low-frequency resonance mode of hematite, ${ }^{1}$ which is relevant to consider for Mössbauer spectroscopy, ${ }^{12}$ we only need to consider the last two terms in Eq. (1). For these two terms and $K_{1}<0, \kappa$ in Eq. (4) is given by ${ }^{36}$

$$
\frac{1}{\kappa}=\frac{1}{2 K_{\mathrm{Bu}}}+\frac{1}{2\left|K_{1}\right|} .
$$

Before we proceed with the detailed modeling of the temperature series of Mössbauer spectra, it is relevant to consider the expected variation of $K_{1}$ with temperature and particle size. For bulk hematite, the temperature variation of $K_{1}$ has been successfully modeled using mean-field theory (see Ref. 1 for references). In this theory, $K_{1}$ attains essentially a constant positive value for temperatures smaller than about $150 \mathrm{~K}$. At higher temperatures it decreases almost linearly with temperature up to about $500 \mathrm{~K} . K_{1}$ changes sign from positive to negative at the Morin transition temperature, $T_{\mathrm{M}}$. For nanoparticles it is known that $T_{\mathrm{M}}$ is smaller than the bulk value and the Morin transition is absent at least down to $5 \mathrm{~K}$ for particles smaller than about $20 \mathrm{~nm}$. ${ }^{1,2,46,47} T_{\mathrm{M}}$ is also sensitive to defects and surface effects. In the study by Bødker et al. ${ }^{12}$ of $16 \mathrm{~nm}$ hematite nanoparticles it was shown that $-K_{1}$ was so large that the sublattice magnetization vectors could be considered as confined to the (111) plane and hence that the $\left|K_{1}\right|^{-1}$ contribution in Eq. (5) could be neglected. As the particles in the present study have a size of about $20 \mathrm{~nm}$, it is likely that $\left|K_{1}\right|$ is small at low temperatures, and hence that the $\left|K_{1}\right|^{-1}$ contribution in Eq. (5) may not be negligible. However, to our best knowledge, there are currently no satisfactory models that predict the temperature dependence of $K_{1}$ for magnetic nanoparticles. Thus, if the temperature variation of $K_{1}$ is similar to that of bulk hematite, we expect that $-K_{1}$ is essentially constant at low temperatures but large above about $150 \mathrm{~K}$. It therefore seems reasonable to include a finite value of $K_{1}$ in the description of the collective magnetic excitations at low temperatures and to consider $K_{1}$ as infinite in a description of the behavior at higher temperatures. As discussed above, it is well known that $K_{1}$ is very sensitive to the particle volume. As a first approximation, we include this in the description of the collective magnetic excitations by assuming that $-K_{1} V$ attains the same value $E_{1}$ for all particle volumes, i.e., that $K_{1}$ is inversely proportional to the particle volume.

The full temperature series of Mössbauer spectra of the coated particles was analyzed using the Blume-Tjon twolevel relaxation model ${ }^{38}$ modified to include the effects of collective magnetic excitations. ${ }^{12,48}$ In this model, the mag- 
netic hyperfine field is assumed to switch with an average frequency $\tau^{-1}$ between $\pm B_{\text {obs }}$, where $B_{\text {obs }}$ is perpendicular to the positive electric field gradient along the [111] axis. The expression, $g\left[v, \tau, B_{\text {obs }}\right]$, for the resulting Mössbauer spectrum for a single particle size, where $v$ is the velocity, can be found in Appendix B of Ref. 38. We have assumed a volume-weighted log-normal distribution, $f_{\ln }(y) d y$ $=(\sqrt{2 \pi} \sigma)^{-1} \exp \left(-\ln ^{2} y / 2 \sigma^{2}\right) d y$, of reduced energy barriers, $y \equiv E_{b} / E_{\mathrm{bm}}=V / V_{\mathrm{m}}$. The expression for a spectrum at a given temperature can then be written as

$$
G(v)=\int_{0}^{\infty} g\left[v, \tau(y), B_{\mathrm{obs}}(y)\right] \cdot f_{\ln }(y) d y,
$$

where $\tau(y)=\tau_{0} \cdot \exp \left(y \cdot E_{\mathrm{bm}} / k T\right)$ and $B_{\mathrm{obs}}(y)$ is the magnetic hyperfine field for a particle with the energy barrier $E_{b}$ $=y \cdot E_{\mathrm{bm}}=y \cdot K_{\mathrm{Bu}} V_{\mathrm{m}}$ and $\left|K_{1}\right| V=E_{1}$. In the fitting, the exact integrals leading to Eqs. (4) and (5) were calculated numerically. Other input parameters not explicitly shown are the quadrupole interaction strength, the isomer shift, and the intrinsic linewidths. The quadrupole interaction strength and the intrinsic linewidths were fixed to be identical for all spectra in the temperature series and the change of the isomer shift with temperature due to the second order Doppler shift was accounted for using the Debye approximation with a Debye temperature of $500 \mathrm{~K} .{ }^{12,49}$ Simultaneous fits of this model to the whole temperature series of Mössbauer spectra were performed for both fixed values of $\tau_{0}$ between $1 \times 10^{-12} \mathrm{~s}$ and $5 \times 10^{-10} \mathrm{~s}$ and for $\tau_{0}$ as a free parameter. The variation of the quality of the fit, $\chi^{2}$, as a function of $\ln \left(\tau_{0}\right)$ had a parabola-like minimum. This variation was used for an estimation of the uncertainties on the parameters from the fit. The uncertainties stated below correspond to the values of $\tau_{0}$ where the value of $\chi^{2}$ has increased $10 \%$ compared to the value at the minimum.

We have fitted the spectra using models for $B_{\text {obs }}$ in which $E_{1}^{-1}=\left(\left|K_{1}\right| V\right)^{-1}$ was assumed negligible (i.e., a pure $2 d$ relaxation) and with $E_{1}$ as a free parameter. The latter model yielded a significantly better fit than the first model, and it thus indicates that the value of $E_{1}$ is relatively small for the particles in the present study.

The best fit is shown as the full lines in Fig. 3(a). We obtained the parameters $\tau_{0}=1.3_{-0.8}^{+1.9} \times 10^{-10} \mathrm{~s}, E_{\mathrm{bm}} / k$ $=K_{\mathrm{Bu}} V_{\mathrm{m}} / k=570 \pm 100 \mathrm{~K}, E_{1} / k=2400_{-700}^{+1600} \mathrm{~K}, \sigma=0.62$ \pm 0.05 and $B_{0}(T=0 \mathrm{~K})=53.4 \pm 0.2 \mathrm{~T}$. The isomer shift extrapolated to zero temperature was $\delta_{0}=0.492 \pm 0.005$ $\mathrm{mm} \mathrm{s}^{-1}$ and the strength of the quadrupole interaction, in terms of the quadrupole shift of the sextet at low temperatures, was $\epsilon=-0.100 \pm 0.005 \mathrm{~mm} \mathrm{~s}^{-1}$. The lower value of $\tau_{0}$ corresponds to the higher value of $E_{\mathrm{bm}}$ and lower value of $E_{1}$.

The corresponding value of $K_{\mathrm{Bu}}$ is about $2 \mathrm{~kJ} \mathrm{~m}^{-3}$. This value is about a factor of 2 lower than the value obtained for the particles with dimensions of about $16 \mathrm{~nm}$ studied by Bødker et al., ${ }^{12}$ and the value of $\tau_{0}$ is about a factor of 2 higher in this study than that obtained by Bødker et al. ${ }^{12}$ The smaller anisotropy may in part be due to a lower crystal stress and hence a smaller stress anisotropy, which is believed to be the predominant contribution to the effective uniaxial anisotropy in the basal plane. ${ }^{1}$ The current models for the relaxation time of a ferromagnetically ordered particle with $3 d$ uniaxial anisotropy predict that $\tau_{0}$, for a constant value of the energy barrier, increases with decreasing anisotropy. ${ }^{50}$ If the behavior of antiferromagnetically ordered particles with $2 d$ uniaxial anisotropy is governed by a similar expression, this may explain the higher observed value of $\tau_{0}$ in the present study, as the median energy barriers in the present study and that by Bødker et al. ${ }^{12}$ are similar, but the anisotropy constant in the present study is lower than that obtained by B $\not \mathrm{dker}$ et al. ${ }^{12}$

\section{Analysis of the spectra of the dry sample}

As discussed in Sec. IV A, the observed slowing down of the superparamagnetic relaxation in the dry sample compared to the coated sample must be due to interparticle interactions. A similar difference between the Mössbauer spectra of wet and dry hematite particles has been observed by Polikarpov et al. ${ }^{7}$ but a detailed investigation of the sample purity, the origin of the interactions and a quantification of the observations was not made by these authors. In this study we have information on both the magnetic moment of the particles and the particle size. This enables us to give a rough estimate of the ordering temperature below which the magnetic dipole interactions lead to freezing of the magnetic moments of the particles.

It has been shown that the ordering temperature of a system of interacting magnetic particles with pure dipole interaction is of the order of

$$
T_{\mathrm{dd}} \equiv \frac{\mu_{0}}{4 \pi} \frac{\mu^{2}}{k D^{3}},
$$

where $\mu$ is the magnetic moment of a particle and $D$ is the average distance between the particles. ${ }^{28}$ For spherical particles of diameter $d$ and mass magnetization $\sigma_{s}$ we can estimate the maximum ordering temperature corresponding to $D$ $=d$ as

$$
T_{\mathrm{dd}}^{\max }=\frac{\mu_{0}}{4 \pi} \frac{\pi^{2} \sigma_{s}^{2} \rho^{2} d^{3}}{36 k} .
$$

Using $d=20 \mathrm{~nm}$ and $\sigma_{s}=0.6 \mathrm{~J} \mathrm{~T}^{-1} \mathrm{~kg}^{-1}$ we estimate $T_{\mathrm{dd}}^{\max } \approx 0.2 \mathrm{~K}$, which is three orders of magnitude lower than the highest temperatures at which we have observed Mössbauer spectra that are still magnetically split. Thus, the freezing of the magnetic moments in the dry sample cannot be explained by magnetic dipole interactions.

For crystallites in close contact, there may be exchange coupling between magnetic surface atoms belonging to neighboring crystallites. This interaction can for a crystallite, $i$, be written as ${ }^{13,36}$

$$
E_{i}=-\vec{M}_{i}(T) \cdot \sum_{j} K_{\mathrm{ex}}^{i j} \vec{M}_{j}(T)
$$

where $\vec{M}_{i}(T)$ and $\vec{M}_{j}(T)$ are one of the sublattice magnetizations in each of the crystallites $i$ and $j$, respectively, and the sum is over the nearest neighbors. Using a mean field approximation, ${ }^{13,36}$ Eq. (9) can be written as

$$
E_{i}=-K_{\mathrm{m}} \vec{M}_{i}(T) \cdot\langle\vec{M}(T)\rangle_{\mathrm{T}},
$$


where $K_{\mathrm{m}}\langle\vec{M}(T)\rangle_{\mathrm{T}}$ is the mean field from the neighboring particles and $K_{\mathrm{m}}=\left\langle\Sigma_{j} K_{\mathrm{ex}}^{i j}\right\rangle$ is an effective exchange coefficient, which depends on the coupling strength and the number of interacting neighbors. We define the order parameter $b(T)$ as

$$
b(T) \equiv\langle M(T)\rangle_{\mathrm{T}} / M_{0}(T),
$$

where $M_{0}(T)$ is the saturation value of the sublattice magnetization at the temperature $T$.

In the analysis of the coated sample, we found that the best description of the data was obtained using a finite value of $K_{1}$ at low temperatures. We also justified that it is probable that $-K_{1}$ attains a large value [i.e., that the sublattice magnetization vectors are confined to the (111) plane] at temperatures above about $150 \mathrm{~K}$. This implies that only the projection of $\langle\vec{M}(T)\rangle_{\mathrm{T}}$ onto the basal plane of particle $i$ is important in Eq. (10). As the sample was obtained after evaporation of the liquid in which the particles were suspended, it is likely that the interparticle interactions have resulted in some local alignment of the orientation of neighboring crystallites due to the easy physical rotation of the particles in the liquid. The gentle crushing of the dry pellet of particles, when the absorber was prepared, may destroy the global but not the local texture of the sample. We therefore assume that $\langle\vec{M}(T)\rangle_{\mathrm{T}}$ is parallel to the easy axis of particle $i$. With this assumption, the total magnetic energy of a particle is given by

$$
\begin{aligned}
E_{\mathrm{tot}}= & -K_{1} V \cos ^{2} \theta+K_{\mathrm{Bu}} V \sin ^{2} \theta \sin ^{2} \phi \\
& -K_{\mathrm{m}} M(T)^{2} b(T) \sin \theta \cos \phi .
\end{aligned}
$$

Using the procedure described in Ref. 36, we find for low temperatures that $\kappa$ in Eq. (4) is given by

$$
\frac{1}{\kappa}=\frac{V}{2\left|K_{1}\right| V+K_{\mathrm{m}} M(T)^{2}}+\frac{V}{2 K_{\mathrm{Bu}} V+K_{\mathrm{m}} M(T)^{2}} .
$$

From the fit shown in Fig. 4, we obtain $\kappa V / k=1400 \pm 150$ $\mathrm{K}$. Using the values of $K_{1} V$ and $K_{\mathrm{Bu}} V$ estimated in Sec. IV B, we find the interaction parameter $K_{\mathrm{m}} M(T)^{2} / k=750$ $\pm 300 \mathrm{~K}$.

At higher temperatures, as discussed above, the total magnetic energy of a particle can be approximated by

$$
E_{\mathrm{tot}} \approx K_{\mathrm{Bu}} V \sin ^{2} \phi-K_{\mathrm{m}} M(T)^{2} b(T) \cos \phi .
$$

With this expression for the energy, we find by use of $2 d$ Boltzmann statistics that

$$
b(T)=\frac{\int_{0}^{\pi} \exp \left[-\beta K_{\mathrm{Bu}} V \sin ^{2} \phi+\beta K_{\mathrm{m}} M(T)^{2} b(T) \cos \phi\right] \cos \phi d \phi}{\int_{0}^{\pi} \exp \left[-\beta K_{\mathrm{Bu}} V \sin ^{2} \phi+\beta K_{\mathrm{m}} M(T)^{2} b(T) \cos \phi\right] d \phi},
$$

where $\beta=(k T)^{-1}$. The temperature dependence of $b(T)$ can be determined from this equation by numerical methods. The temperature at which $b(T)$ becomes zero is called the ordering temperature, $T_{\mathrm{p}}$. For the case of zero anisotropy, $K_{\mathrm{Bu}}$ $=0$, we obtain the equation

$$
b^{0}(T)=\frac{I_{1}\left(\beta K_{\mathrm{m}} M(T)^{2} b^{0}(T)\right)}{I_{0}\left(\beta K_{\mathrm{m}} M(T)^{2} b^{0}(T)\right)},
$$

where $I_{n}(x)$ is the modified Bessel function of the first kind of order $n$. Using the expansions, $I_{0}(x) \approx 1+x^{2} / 4$ and $I_{1}(x)$ $\approx x / 2$ for $x \ll 1$, it is easy to derive the ordering temperature for $K_{\mathrm{Bu}}=0$ as

$$
T_{\mathrm{p}}^{0}=K_{\mathrm{m}} M\left(T_{\mathrm{p}}^{0}\right)^{2} / 2 k .
$$

It is convenient to express the interaction strength in terms of $T_{\mathrm{p}}^{0}$ instead of $K_{\mathrm{m}} M(T)^{2}$. This is easily carried out in Eqs. (15) and (16) by making the replacement

$$
\beta K_{\mathrm{m}} M(T)^{2}=\frac{2 T_{\mathrm{p}}^{0}}{T}\left(M(T) / M\left(T_{\mathrm{p}}^{0}\right)\right)^{2} .
$$

The effect of the anisotropy is to enhance the ordering temperature $T_{\mathrm{p}}$ with respect to $T_{\mathrm{p}}^{0}$ and change the curvature of $b(T)$ compared to $b_{0}(T) .^{51}$
In the "super-ferromagnetism" model, it is assumed that the superparamagnetic relaxation is fast, i.e., the Mössbauer spectrum of a single particle in principle should consist of a sextet with narrow lines. The magnetic splitting is determined by the average hyperfine field, which is proportional to $b(T)$ depends on the interaction strength and the temperature. The random packing in typical samples of nanoparticles will, however, result in variations in the interaction strength leading to a distribution of magnetic hyperfine fields at finite temperatures.

It should be noticed that if the interaction energy is large compared to the anisotropy energy, there is only one energy minimum of the magnetic energy [Eq. (14)]. In this case, there is no energy barrier to overcome in the relaxation process. The magnetization directions are then expected to fluctuate between different precession states with a relaxation time of the order of $\tau_{0}$, which is short compared to the time scale of Mössbauer spectroscopy.

In accordance with the model for "superferromagnetism," the spectra were fitted with distributions of magnetic hyperfine fields using the method by Wivel and Mbrup. ${ }^{37}$ The results for selected temperatures are shown in Fig. 5. A doublet appears in such distributions as a sextet with a magnetic hyperfine field close to zero. Even at $323 \mathrm{~K}$, there is essentially no doublet but there is a very broad distribution of magnetic hyperfine fields. 


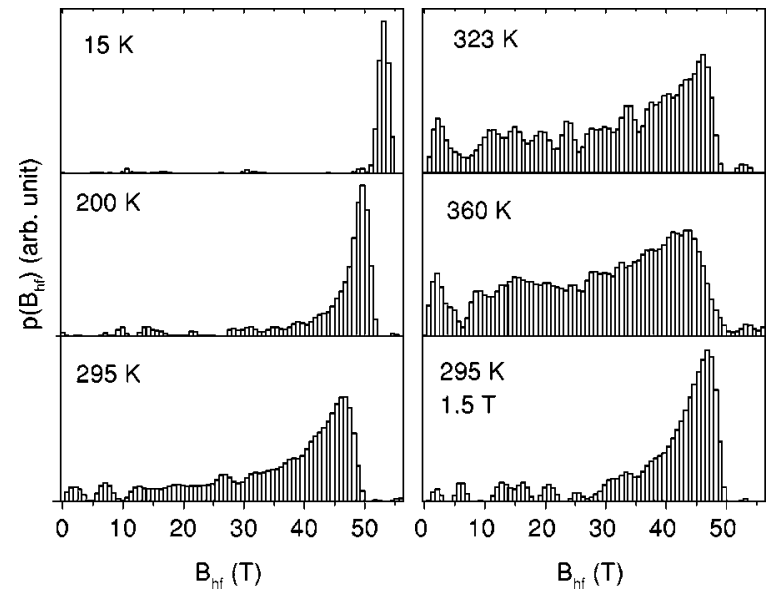

FIG. 5. Representative distributions of hyperfine fields for the spectra of the dry sample obtained at the indicated temperatures and fields. The distributions obtained at $295 \mathrm{~K}$ are shown on the same scale.

In earlier publications on interacting goethite particles, ${ }^{13,14}$ the order parameter was fitted to the reduced average hyperfine field

$$
b_{\mathrm{av}}(T)=\left\langle B_{\mathrm{hf}}(T)\right\rangle / B_{0}(T),
$$

where $\left\langle B_{\mathrm{hf}}(T)\right\rangle$ was obtained from the distribution of hyperfine fields. The saturation hyperfine field at zero temperature was a fitting parameter in the fits to $b(T)$ and the temperature variation of the saturation hyperfine field, $B_{0}(T)$, was assumed to follow that of bulk hematite after subtracting 0.8 T below the Morin transition. ${ }^{1}$ In Fig. 6 is shown $b_{\text {av }}(T)$, deduced from fits of the spectra of the dry sample to hyperfine field distributions, as a function of temperature. Results are only given for $T \leqslant 323 \mathrm{~K}$ where essentially no doublet is present in the spectra. Higher temperatures may also lead to removal of adsorbed water from the particle surfaces, which may change the coupling between the particles. Fits to the data of the "super-ferromagnetic" order parameter with and without including the anisotropy are shown as the full and dashed lines, respectively. From the fit in which the anisotropy is neglected we obtain $T_{\mathrm{p}}^{0}=395 \pm 5 \mathrm{~K}$. When the anisotropy is included we obtain $T_{\mathrm{p}}=382 \pm 5 \mathrm{~K}$ corresponding to

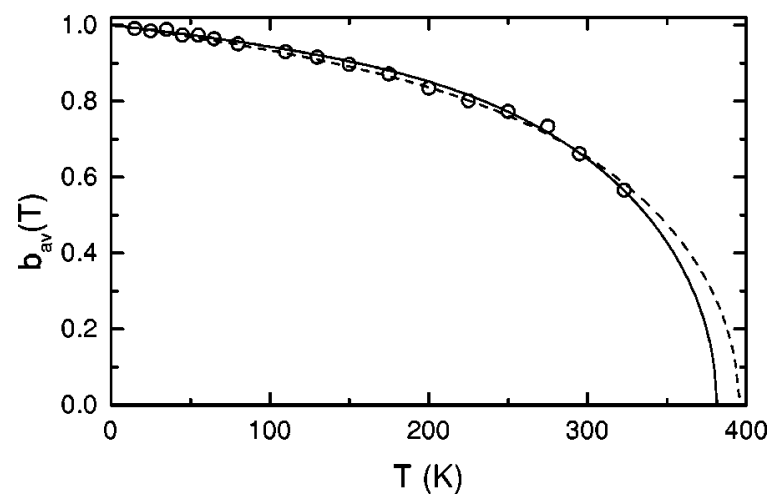

FIG. 6. Values of $b_{\mathrm{av}}(T)$ obtained from distribution fits of the spectra of the dry sample as described in the text. The solid and broken lines are fits to the data of $b(T)$ and $b_{0}(T)$ obtained from Eqs. (14) and (15), respectively.

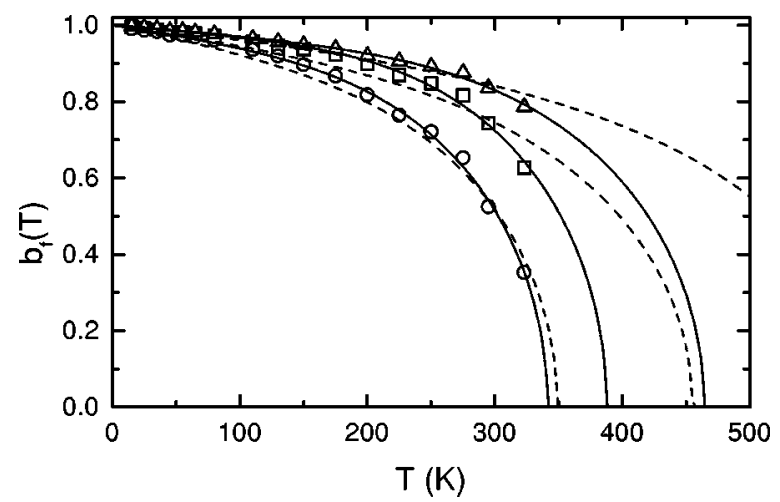

FIG. 7. Values of $b_{f}(T)$ obtained as described in the text for the dry sample as a function of temperature for $f=0.25(\bigcirc), 0.5(\square)$, and $0.75(\triangle)$. The solid and broken lines are best fits of $b(T)$ and $b_{0}(T)$ to the data, respectively.

$T_{\mathrm{p}}^{0}=370 \pm 20 \mathrm{~K}$ and $K_{\mathrm{Bu}} V / k=90 \pm 90 \mathrm{~K}$. Thus, it is seen that the best fits are obtained for an anisotropy close to zero. This rather puzzling observation was also made in earlier studies performed on goethite nanoparticles. ${ }^{13,14}$

The average hyperfine field is very sensitive to a small fraction of superparamagnetic particles and does effectively not only depend on the averaging over the interaction fields but also on an averaging over the anisotropy energies. It is therefore possible that the effect of the anisotropy is averaged out when the average hyperfine field is considered. It would therefore be more appropriate to consider the temperature dependence of a fraction of the particles with the same interaction energies and anisotropy energies. The order parameter of each particle in the sample is assumed to be given by an expression of the same form as Eq. (15). In the following we assume that the shape of the individual $b(T)$ curves are similar, but with different values of $T_{\mathrm{p}}$, i.e., we assume that the curves do not intersect. The temperature dependence of the order parameter for particles with similar behavior can then be obtained from the hyperfine field distributions (Fig. 5). We define the order parameter $b_{f}(T)$ as

$$
b_{f}(T)=B_{f}(T) / B_{0}(T),
$$

where $B_{f}(T)$ is the $f$-quantile of the hyperfine field distribution $p\left(B_{\mathrm{hf}}(T)\right)$, i.e.,

$$
f=\int_{0}^{B_{f}(T)} p\left(B_{\mathrm{hf}}(T)\right) d B_{\mathrm{hf}}(T) .
$$

In Fig. 7 is shown the variation of $b_{f}(T)$ for $f=0.25,0.5$, and 0.75 along with fits to the "super-ferromagnetic" order parameter with and without the anisotropy included [Eqs. (15) and (16), respectively]. It is seen that the quality of the fits for which the anisotropy is not included is poor, while the quality of the fits with the anisotropy included is rather good. The difference in the quality of the fits is most clearly seen for $f=0.5$ and 0.75 . In the following we only consider the fits to the model where the anisotropy is included in the calculations.

In Fig. 8 are shown the interaction strength, $T_{\mathrm{p}}^{0}$, and $K_{\mathrm{Bu}} V / k$ obtained from fits of $b(T)$ to the experimental values of $b_{f}(T)$. The $2 d$ relaxation model [Eq. (15)] is not strictly applicable at low temperatures, where $\left|K_{1}\right|^{-1}$ is not 


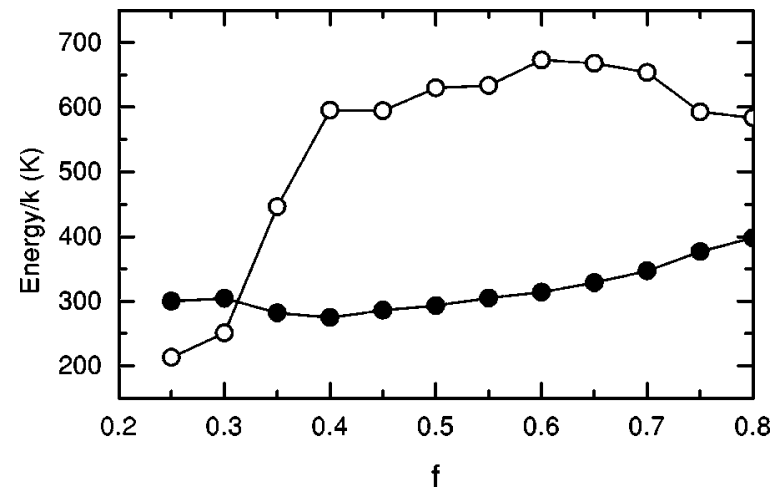

FIG. 8. Values of $T_{\mathrm{p}}^{0}(\bigcirc)$ and $K_{\mathrm{Bu}} V / k(\bigcirc)$ obtained from fits of $b(T)$ to $b_{f}(T)$ determined from the hyperfine field distributions for the dry sample [Fig. 4(b)].

negligible. However, the experimentally observed variation of $b(T)$ at low temperatures is small compared to that at higher temperatures, and the influence of a finite value of $K_{1}$ at low temperatures on the estimates of $T_{\mathrm{p}}^{0}$ and $K_{\mathrm{Bu}} V / k$ is therefore negligible.

For $f>0.4$, the value of $K_{\mathrm{Bu}} V / k$ is about $600 \mathrm{~K}$ and $T_{\mathrm{p}}^{0}$ is of the order $300-400 \mathrm{~K}$. Thus, $K_{\mathrm{Bu}} V / k \simeq K_{\mathrm{m}} M(T)^{2} b(T) / k$ in the considered temperature range. If the two parameters are identical $(=600 \mathrm{~K})$, the magnetic energy [Eq. (14)] will have two minima, but the probability of finding a particle in the upper minimum (at $\phi=\pi$ ) is less than $2 \%$ at $300 \mathrm{~K}$ and even smaller at low temperatures. It is therefore a good approximation to assume that the relaxation takes place in a single minimum and therefore, even at low temperatures, the relaxation is fast compared to the time scale of Mössbauer spectroscopy.

The values of $K_{\mathrm{Bu}} V$, obtained from the fits, are significantly smaller for $f \leqslant 0.4$ than for $f \geqslant 0.4$. It is unlikely that the assumption that the mean field for neighboring particles is parallel to the easy direction of magnetization is fulfilled for all particles in the sample and this may explain the irregular behavior for $f \leqslant 0.4$. As discussed above, only the projection of $\langle\vec{M}(T)\rangle_{\mathrm{T}}$ onto the basal plane contributes to the effective interaction strength and therefore particles with a large angle between the easy direction and $\langle\vec{M}(T)\rangle_{\mathrm{T}}$ will often have low values of $T_{\mathrm{p}}$. Moreover, the shape of the $b(T)$ curves depends on this angle. For particles with simple uniaxial anisotropy and a large angle between $\langle\vec{M}(T)\rangle_{\mathrm{T}}$, the anisotropy even leads to a reduction of $T_{\mathrm{p}}$ compared to $T_{\mathrm{p}}^{0}$ instead of the increase found for small angles. ${ }^{51}$ Fits to the $b_{f}(T)$ data to Eq. (15) are therefore not justified for particles for which this angle is large. Such particles will predominantly contribute to the lower part of the distribution of magnetic hyperfine fields. In the following, we therefore only discuss the results obtained for $f \geqslant 0.4$.

The average value of $K_{\mathrm{Bu}} V / k$ is about $650 \mathrm{~K}$ and the average value of $T_{\mathrm{p}}^{0}$ is about $300 \mathrm{~K}$. The value of $K_{\mathrm{Bu}} V / k$ is in excellent agreement with that obtained from the analysis of the temperature series of Mössbauer spectra of the coated sample, and the value of $T_{\mathrm{p}}^{0}$ is in accordance with the value of the interaction parameter estimated from Eq. (13). In the range of $f$ values from about 0.4 to 0.75 , where we expect to have reliable values of $b_{f}(T)$, a slight increase of both $T_{\mathrm{p}}^{0}$ and $K_{\mathrm{Bu}} V / k$ with increasing $f$ is observed. This is in agreement with the assumption that the larger values of $f$ correspond to particles with larger anisotropy energies and stronger interactions. Hence, the analysis of the temperature variation of the hyperfine fields corresponding to fixed fractions of the material appears to give information about the physical properties of the sample, whereas the analysis of the variation of the average hyperfine field leads to low and unreliable values for the magnetic anisotropy and a slight overestimation of the interaction strength, $T_{\mathrm{p}}^{0}$, due to an averaging over the distribution of anisotropy energy barriers and the distribution of interaction strengths. This result seems to explain why the best fits to the model for "superferromagnetism", were obtained for zero anisotropy for samples of goethite in Refs. 13 and 14.

The present study has the advantage that samples of virtually noninteracting particles and interacting particles, prepared from the same batch of particles, could be compared. Moreover, the particles have a nonzero magnetic moment, which can be aligned along an external magnetic field such that we unambiguously can distinguish between paramagnetic and superparamagnetic behavior. Furthermore, the Néel temperature of hematite $\left(T_{\mathrm{N}} \approx 956 \mathrm{~K}\right)$ is much larger than the Néel temperature of goethite $\left(T_{\mathrm{N}} \approx 393 \mathrm{~K}\right)$ and this excludes the possibility of a distribution of Néel temperatures near room temperature. The data are in excellent agreement with the "super-ferromagnetism" model and this model therefore seems to be able to explain the essential features of ordering phenomena due to exchange interactions between antiferromagnetic or weakly ferromagnetic nanoparticles.

Since the dipole interactions between the hematite particles are too weak to explain the ordering it seems that the exchange coupling between atoms belonging to neighboring particles is responsible for the ordering. For a pair of particles it is likely that only a few pairs of atoms can be in so close contact that the exchange coupling is significant. It is possible to make a rough estimate of the number of atom pairs necessary to explain the observed ordering temperature. From the ordering temperature $T_{\mathrm{p}}^{0} \approx 300 \mathrm{~K}$ we find from Eq. (17) that the interaction energy for a particle at $T=0 \mathrm{~K}$ is about $\left|E_{i} / k\right| \approx 600 \mathrm{~K}$. The dominating contribution to the exchange coupling between the iron atoms in bulk hematite comes from the superexchange coupling of each iron atom to nine neighboring iron atoms. ${ }^{1}$ Reported exchange coupling constants, $J / k$, for the superexchange pathways range from about $-10 \mathrm{~K}$ to $-30 \mathrm{~K}$. ${ }^{1}$ The value $J / k \approx-20 \mathrm{~K}$ gives an exchange energy for a pair of $\mathrm{Fe}^{3+}$ ions $(s=5 / 2)$ of about $-J s^{2} / k \approx 125 \mathrm{~K}$. Hence, if a particle is connected to the neighboring particles via only about 5 exchange bridges with the same exchange coupling constants as inside the particles we can explain the observed ordering temperature. It is, however, likely that more pairs of atoms are involved in the exchange coupling and that most of these have a weaker exchange coupling constant.

The particles have been prepared in water and water molecules are therefore adsorbed onto the surface. Such molecules may prevent direct contact between the surface atoms of the neighboring particles, but they may provide another exchange pathway in which the coupling strength may resemble that of iron atoms in hydrated iron salts. Such compounds typically have magnetic ordering temperatures of the 
order of $1 \mathrm{~K}$ and exchange coupling constants of about $|J / k| \approx 0.1 \mathrm{~K}$. In this case we find that about 1000 coupled pairs of atoms are needed to account for the interaction strength. Assuming a surface layer thickness of $0.2 \mathrm{~nm}$, we find that a spherical particle with a diameter of $20 \mathrm{~nm}$ contains about 5000 iron atoms in the surface layer. This implies that even for this weak coupling strength only a small fraction of the surface atoms is needed to form exchange bridges through water to the iron atoms in the surfaces of neighboring particles to explain the observed ordering temperature.

\section{CONCLUSIONS}

We have shown that the described preparation procedure leads to formation of hematite nanoparticles with dimensions of about $20 \mathrm{~nm}$ and that coprecipitated ferrihydrite can be removed by treatment with oxalate. From the same batch of particles a coated sample with negligible interparticle interactions and a dry sample with strong interparticle interactions have been prepared.

The magnetization of the coated particles is similar to that of bulk hematite. By simultaneous fitting of a temperature series of Mössbauer spectra to the described modified Blume-Tjon model we have estimated the energy barrier in the basal plane, $K_{\mathrm{Bu}} V_{\mathrm{m}} / k=570 \pm 100 \mathrm{~K}$, the low-temperature out-of-plane anisotropy energy, $E_{1} / k=2400_{-700}^{+1600} \mathrm{~K}$, and the pre-exponential factor, $\tau_{0}$ $=1.3_{-0.8}^{+1.9} \times 10^{-10} \mathrm{~s}$.
The values of $K_{\mathrm{Bu}}$ and $\tau_{0}$, obtained in this study, are smaller and larger, respectively, than those obtained for smaller hematite nanoparticles prepared by a different method. ${ }^{12}$ The lower value of $K_{\mathrm{Bu}}$ may be due to a lower degree of stress in the particles in the present study. The higher value of $\tau_{0}$ may be explained by a dependence of $\tau_{0}$ on the anisotropy, provided that the superparamagnetic relaxation time for an antiferromagnet in two dimensions is given by an expression similar to that of a ferromagnet in three dimensions.

It has been shown that the shape of the spectra of the dry sample at high temperatures is due to strong interparticle interactions and that the dominating contribution to the interactions comes from exchange coupling of the surface atoms of neighboring particles. The effect of the interactions and the magnetic anisotropy on the distribution of hyperfine fields can be consistently analyzed using a two-dimensional mean field model for the interactions similar to the model for "super-ferromagnetism" by Mbrup et al. ${ }^{13}$ if the variation of the hyperfine field corresponding to a certain fraction of the material and not the average hyperfine field is considered.

\section{ACKNOWLEDGMENTS}

Financial support from the Danish Natural Science Research Council and the Danish Technical Research Council is gratefully acknowledged.
${ }^{1}$ A. H. Morrish, Canted Antiferromagnetism: Hematite (World Scientific, Singapore, 1994).

${ }^{2}$ W. Kündig, H. Bömmel, G. Constabaris, and R. H. Lindquist, Phys. Rev. 142, 327 (1966).

${ }^{3}$ A. M. van der Kraan, Phys. Status Solidi A 18, 215 (1973).

${ }^{4}$ D. G. Rancourt, S. R. Julian, and J. M. Daniels, J. Magn. Magn. Mater. 49, 305 (1985).

${ }^{5}$ P. Ayyub, M. Multani, M. Barma, V. R. Palkar, and R. Vijayaraghavan, J. Phys. C 21, 2229 (1988)

${ }^{6}$ J. Jing, F. Zhao, X. Yang, and U. Gonser, Hyperfine Interact. 54, 571 (1990).

${ }^{7}$ M. A. Polikarpov, I. V. Trushin, and S. S. Yakimov, J. Magn. Magn. Mater. 116, 372 (1992).

${ }^{8}$ R. Zysler, D. Fiorani, J. L. Dormann, and A. M. Testa, J. Magn. Magn. Mater. 133, 71 (1994).

${ }^{9}$ M. F. Hansen, F. Bødker, S. Mørup, K. Lefmann, K. N. Clausen, and P.-A. Lindgård, Phys. Rev. Lett. 79, 4910 (1997).

${ }^{10}$ M. Vasquez-Mansilla, R. D. Zysler, C. Arciprete, M. I. Dimitrijewits, C. Saragovi, and J. M. Greneche, J. Magn. Magn. Mater. 204, 29 (1999).

${ }^{11}$ R.A. Borzi, S. J. Stewart, G. Punte, R. C. Mercader, M. VasquezMansilla, R. D. Zysler, and E. D. Cabanillas, J. Magn. Magn. Mater. 205, 234 (1999).

${ }^{12}$ F. Bødker, M. F. Hansen, C. Bender Koch, K. Lefmann, and S. Mbrup, Phys. Rev. B 61, 6826 (2000).

${ }^{13}$ S. Mørup, M. B. Madsen, J. Franck, J. Villadsen, and C. J. W. Koch, J. Magn. Magn. Mater. 40, 163 (1983).

${ }^{14}$ C. J. W. Koch, M. B. Madsen, S. Mørup, G. Christiansen, and L. Gerward, Clays Clay Miner. 34, 17 (1986).
${ }^{15}$ S. Mbrup, P. H. Christensen, and B. S. Clausen, J. Magn. Magn. Mater. 68, 160 (1987).

${ }^{16}$ S. Mørup, Hyperfine Interact. 60, 959 (1990).

${ }^{17}$ S. Mørup, Hyperfine Interact. 90, 583 (1994).

${ }^{18}$ C. Bender Koch, C. A. Oxborrow, S. Mørup, M. B. Madsen, A. J. Quinn, and J. M. D. Coey, Phys. Chem. Miner. 22, 333 (1995).

${ }^{19}$ J. L. Dormann, L. Bessais, and D. Fiorani, J. Phys. C 21, 2015 (1988).

${ }^{20}$ S. Mørup, Europhys. Lett. 28, 671 (1994).

${ }^{21}$ S. Mørup, F. Bødker, P. V. Hendriksen, and S. Linderoth, Phys. Rev. B 52, 287 (1995).

${ }^{22}$ J. Zhang, C. Boyd, and W. Luo, Phys. Rev. Lett. 77, 390 (1996).

${ }^{23}$ J. L. Dormann, D. Fiorani, and E. Tronc, Adv. Chem. Phys. 98, 283 (1997)

${ }^{24}$ C. Djurberg, P. Svedlindh, P. Nordblad, M. F. Hansen, F. B ddker, and S. Mørup, Phys. Rev. Lett. 79, 5154 (1997).

${ }^{25}$ T. Jonsson, P. Svedlindh, and M. F. Hansen, Phys. Rev. Lett. 81, 3976 (1998).

${ }^{26}$ H. Mamiya, I. Nakatani, and T. Furubayashi, Phys. Rev. Lett. 80, 177 (1998).

${ }^{27}$ J. L. Dormann, D. Fiorani, and E. Tronc, J. Magn. Magn. Mater. 202, 251 (1999).

${ }^{28}$ M. F. Hansen and S. Mørup, J. Magn. Magn. Mater. 184, 262 (1998).

${ }^{29}$ J. L. Dormann, D. Fiorani, R. Cherkaoui, E. Tronc, F. Lucari, F. D’Orazio, L. Spinu, M. Nogués, H. Kachkachi, and J.P. Jolivet, J. Magn. Magn. Mater. 203, 23 (1999).

${ }^{30}$ D. Fiorani, J. L. Dormann, R. Cherkaoui, E. Tronc, F. Lucari, F. D’Orazio, L. Spinu, M. Nogués, A. Garcia, and A. M. Testa, J. 
Magn. Magn. Mater. 196-197, 143 (1999).

${ }^{31}$ U. Schwertmann, Z. Pflanzenernähr, Dueng., Bodenkd. 105, 194 (1964).

${ }^{32}$ R. M. Cornell and U. Schwertmann, The Iron Oxides $(\mathrm{VCH}$, Weinheim, Germany, 1996).

${ }^{33}$ See, for example, B. E. Warren, X-Ray Diffraction (AddisonWesley, Reading, MA, 1969).

${ }^{34}$ E. Murad and U. Schwertmann, Am. Mineral. 65, 1044 (1980).

${ }^{35}$ R. J. Pollard, C. M. Cardile, D. G. Lewis, and L. J. Brown, Clay Miner. 27, 57 (1992).

${ }^{36}$ S. Mørup, J. Magn. Magn. Mater. 37, 39 (1983).

${ }^{37}$ C. Wivel and S. Mørup, J. Phys. E 14, 605 (1981).

${ }^{38}$ M. Blume and J. A. Tjon, Phys. Rev. 165, 446 (1968).

${ }^{39}$ A. Govaert, C. Dauwe, E. de Grave, and J. de Sitter, Solid State Commun. 18, 389 (1976).

${ }^{40}$ M. B. Madsen, S. Mørup, and C. J. W. Koch, Hyperfine Interact. 42, 1059 (1988).

${ }^{41}$ S. Bocquet and S. J. Kennedy, J. Magn. Magn. Mater. 109, 260 (1992).
${ }^{42}$ S. Bocquet, R. J. Pollard, and J. D. Cashion, Phys. Rev. B 46, 11 657 (1992)

${ }^{43}$ J. M. D. Coey, A. Barry, J.-M. Brotto, H. Rakoto, W. N. Mussel, A. Collomb, and D. Fruchard, J. Phys.: Condens. Matter 7, 759 (1995).

${ }^{44}$ S. Mørup, Surf. Sci. 156, 888 (1985).

${ }^{45}$ C. Hunt, Q. A. Pankhurst, and D. P. E. Dickson, Hyperfine Interact. 91, 821 (1994).

${ }^{46}$ D. Schroer and R. C. Nininger, Jr., Phys. Rev. Lett. 19, 632 (1967).

${ }^{47}$ R. C. Nininger, Jr. and D. Schroer, J. Phys. Chem. Solids 39, 137 (1978).

${ }^{48}$ S. Mbrup, H. Topsøe, and J. Lipka, J. Phys. Colloq. 37, C6-287 (1976).

${ }^{49}$ V. G. Bhide, Mössbauer Effect and its Applications (Tata McGraw-Hill Publishing Co., New Delhi, 1973), p. 93.

${ }^{50}$ W. T. Coffey, P. J. Cregg, and Yu P. Kalmykov, Adv. Chem. Phys. 83, 263 (1993).

${ }^{51}$ S. Mørup and G. Christiansen, J. Appl. Phys. 73, 6955 (1993). 